\title{
Functional Consequences of the Asymmetric Architecture of the Ctenophore Statocyst
}

\author{
SIDNEY L. TAMM* \\ Bell Center, Marine Biological Laboratory, Woods Hole, Massachusetts 02543
}

\begin{abstract}
Ctenophores, or comb jellies, are geotactic with a statocyst that controls the activity of the eight ciliary comb rows. If a ctenophore is tilted or displaced from a position of vertical balance, it rights itself by asymmetric frequencies of beating on the uppermost and lowermost comb rows, turning to swim up or down depending on its mood. I recently discovered that the statocyst of ctenophores has an asymmetric architecture related to the sagittal and tentacular planes along the oral-aboral axis. The four groups of pacemaker balancer cilia are arranged in a rectangle along the tentacular plane, and support a superellipsoidal statolith elongated in the tentacular plane. By controlled tilting of immobilized ctenophores in either body plane with video recording of activated comb rows, I found that higher beat frequencies occurred in the sagittal than in the tentacular plane at orthogonal orientations. Similar tilting experiments on isolated statocyst slices showed that statolith displacement due to gravity and the resulting deflection of the mechanoresponsive balancers are greater in the sagittal plane. Finally, tilting experiments on a mechanical model gave results similar to those of real statocysts, indicating that the geometric asymmetries of statolith design are sufficient to account for my findings. The asymmetric architecture of the ctenophore statocyst thus has functional consequences, but a possible adaptive value is not known.
\end{abstract}

\section{Introduction}

Francis Crick once said, "If you want to understand function, study structure" (Crick, 1988). The statocyst of ctenophores is a long-studied structure whose function is generally understood. Recent findings, however, show that the design of the structure is different from what was supposed. This report investigates whether the newly re-

Received 15 March 2015; accepted 28 May 2015.

* E-mail address: tamm@bu.edu vised structure affects its function. Specifically, does the recently discovered asymmetric architecture of the ctenophore statocyst, with respect to the sagittal and tentacular body planes (Tamm, 2014c), influence its functional responses during geotaxis?

Ctenophores, or comb jellies, are among the most beautiful of the marine zooplankton, with iridescent waves of color coursing along their ciliary rows. They are the largest animals to use cilia for locomotion, and the longest cilia in nature are found in the eight rows of locomotory comb plates of ctenophores. The large cilia in the comb plates and in the macrocilia of ctenophores offer experimental advantages for studying motile and sensory functions of cilia (Tamm, 1980, 1982, 2014a, b), and ciliary development and regeneration (Tamm, 2012a, b).

Ctenophores have an equilibrium receptor system, or statocyst, that uses the gravitational field as a reference (Horridge, 1966, 1971, 1974; Budelmann, 1988). Ctenophores often assume a vertical position in the water with their major longitudinal (oral-aboral) axis parallel to the direction of gravity: either feeding mouth up at the surface, or swimming mouth downward, away from the surface (Tamm, 1982, 2014a). If a ctenophore is tilted or displaced from either position of vertical balance, it rights itself by asymmetric frequencies of comb plate beating on the uppermost and lowermost comb rows (Horridge, 1974). The lower comb rows beat faster than the upper rows, with ciliary power strokes directed aborally, when the animal turns to swim mouth upward (negative geotaxis). The converse occurs during downward steering (positive geotaxis). After the animal regains a vertical orientation, all comb rows beat at a similar frequency. The tendency to swim up or down (the sign of geotaxis) is called a mood, and is influenced by environmental and perhaps endogenous stimuli (Horridge, 1966, 1971, 1974; Tamm, 1982, 2014a). 
The statocyst resides in the aboral sense organ, or apical organ, at the aboral pole of the body. The ctenophore apical organ is structurally and functionally unique in the animal kingdom (Tamm, 2014a); and it contains other sensory elements besides the statocyst, including putative photoreceptor cells that express opsins (Schnitzler et al., 2012) and pressure receptors (Tamm, 1982), with possible influences on geotactic mood. The single large statolith consists of numerous aggregated living cells, or lithocytes. Each lithocyte is filled with a membrane-bound calcareous concretion surrounded by a rim of cytoplasm and nucleus (Samassa, 1892; Krisch, 1973; Aronova, 1974; Tamm, 1982, 2014a, c; Noda and Tamm, 2014). The statolith is supported upon the ends of four sickle-shaped groups of cilia called balancers, each located in a body quadrant and comprising 150-200 mechanoresponsive cilia. The balancers are motile and act as pacemakers for beating of the comb rows (Chun, 1880; Horridge, 1966, 1971, 1974; Tamm, 1982, 2014a). Each beat of a balancer is propagated mechanically as a wave of beating along its two narrow tracts of short cilia (ciliated grooves) to a pair of comb rows in that quadrant of the body. The beat frequency of a balancer depends on the amount it is bent by the gravitational load of the statolith and the direction of bending (Tamm, 1982, 2014a). The same mechanical stimulus can either excite or inhibit beating of a balancer and the two comb rows connected to it, depending on the ctenophore's sign of geotaxis, or mood (Tamm, 1982, 2014a; Lowe, 1997). The balancers thus serve as both mechanoreceptors and direct effectors to control swimming direction and orientation with respect to gravity (Horridge, 1966, 1971, 1974; Tamm, 1982, 2014a).

Ctenophores display features of bilateral symmetry around the oral-aboral axis: the flattened stomodeum defines the sagittal plane, and an orthogonal tentacular plane passes through the two tentacle pouches. Lobate ctenophores such as Mnemiopsis are compressed in the tentacular plane and expanded in the sagittal plane, with two oral lobes on either side of the mouth (Fig. 1). The four comb rows bordering the sagittal plane (subsagittal rows) are longer and have more comb plates than the four shorter comb rows bordering the tentacular plane (subtentacular rows).

I recently discovered that the statolith of Mnemiopsis, Pleurobrachia, and Beroe is not a spherical mass of lithocytes, as previously supposed, but has a superellipsoidal shape with its major axis parallel to the tentacular plane (Fig. 1) (Tamm, 2014c). This non-radial symmetry of the statolith is due to two factors: a rectangular arrangement of the four balancers, which arise at the vertices of a rectangle with a 3:1 length-width ratio oriented along the tentacular plane (Fig. 1) (Tamm, 2014c); and the addition of new lithocytes only at the two ends of the statolith by transport along the ciliary surfaces of the balancers (Noda and Tamm, 2014).

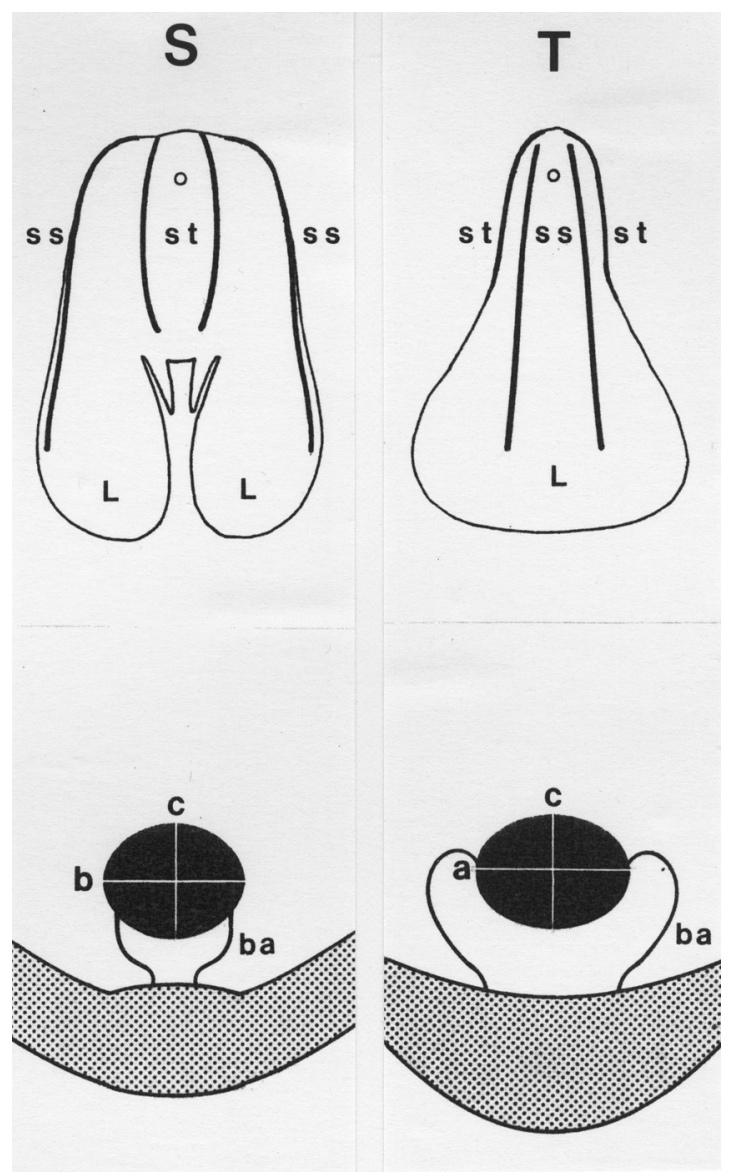

Figure 1. Diagram of Mnemiopsis (top panels) and higher magnification of its statocyst (lower panels), as viewed from the side in the sagittal plane ( $\mathrm{S}$, left column) and in the tentacular plane ( $\mathrm{T}$, right column). The statocyst is located within the small open circle near the aboral end of the animal (top). Two rounded lobes (L) project from the oral end. The longer subsagittal comb rows (ss) and the shorter subtentacular comb rows (st) are shown by heavy lines running parallel to the aboral-oral axis (vertical). The major (a), minor (b), and depth (c) axes of the superellipsoidal statolith (black) and the rectangular arrangement of the four balancers (ba) on the epithelial floor (stippled) are oriented orthogonally in the two body planes. Note that the longer a-axis of the statolith and the greater spacing between balancer pairs occurs along the tentacular plane. (Modified from Tamm, 2014c).

My aim was to discover whether these bilateral asymmetries in statocyst architecture affect the function of the statocyst during geotactic behavior of ctenophores.

\section{Materials and Methods}

\section{Organisms}

Mnemiopsis leidyi A. Agassiz, 1865, were carefully dipped from the surface of the sea at Woods Hole, Massachusetts. Freshly collected animals were used immediately or kept briefly in flowing seawater at the Marine Biological Laboratory (Woods Hole, MA). 


\section{Whole-animal tilting}

Adult Mnemiopsis (3.5 cm average body length along the oral-aboral axis) were individually mounted with two insect pins on a 9-cm diameter transparent Sylgard 184 (Dow Corning) disk. The center of the disk was attached to a glass rod axle inserted through a Lucite support tray. The disk could be manually rotated in a vertical plane to different orientations with respect to gravity in a Lucite viewing box filled with seawater (cteno-tilter; Fig. 2) (Tamm, 1980, 1982). Each animal was pinned first in either the sagittal or tentacular body plane. The beat frequencies of comb rows on opposite sides of the body, viewed in profile from the front of the cteno-tilter, were recorded by a video camera at four orientations to gravity in the following sequence: the oral-aboral axis vertical with the aboral pole facing up $\left(0^{\circ}\right)$, the oral-aboral axis horizontal with the aboral pole facing to the right $\left(90^{\circ}\right)$, the oral-aboral axis vertical with the aboral pole facing down $\left(180^{\circ}\right)$, and the oral-aboral axis horizontal with the aboral pole facing to the left $\left(270^{\circ}\right)$.

Two horizontal tilt positions, at $90^{\circ}$ and $270^{\circ}$, were chosen because the differences in beat frequencies between the uppermost and lowermost comb rows are graded with tilt angle and reach a maximum at $45^{\circ}$ through $90^{\circ}$ deviation from vertical (Tamm, 1980, 1982). Right-angle orientations of the animal also were more convenient to select.

After testing in a given body plane (sagittal or tentacular), each animal was unpinned and remounted on the disk in the other mutually perpendicular plane (tentacular or sagittal) for a second round of testing at the same orientations $\left(0^{\circ}\right.$, $90^{\circ}, 180^{\circ}$, and $270^{\circ}$ ). The body plane used first for testing was alternated in successive animals to control for possible effects on beat frequencies of time and repeated pinning in the cteno-tilter.

The activity of comb rows on opposite sides of the body was recorded for $1-1.5 \mathrm{~min}$ at each body orientation. This cycle of tilting and recording was usually repeated, at least for the two horizontal orientations at $90^{\circ}$ and $270^{\circ}$. Activation or inhibition of beating at a given orientation was often uniform during the recording period, but sometimes included temporary slowdowns or pauses. Measurements of beat frequency (see next section) were made only during continuous activity.

\section{Beat frequency recording}

The activity of comb rows of pinned animals was recorded by a Zeiss ZVS-3C75DE video camera and Nikon Micro-NIKKOR $55 \mathrm{~mm}$ 1:2.8 lens aimed at the front wall of the transparent, back-illuminated cteno-tilter (normal to the Sylgard disk and plane of tilting) (Fig. 2). A camera exposure time of $0.5 \mathrm{~ms}$ or $1 \mathrm{~ms}$ gave sharp, still-field images of successive stages in the comb plate beat cycle. Images and audio tracks of relevant information were recorded on SVHS videocassettes with a Panasonic AG-7355 VCR that allowed field-by-field playback (16.7 ms per field), coupled to a QSI-VFF 6030 field/frame counter and video monitor.

\section{Beat frequency analysis}

Changes in geotactic sign are often accompanied by variations in beat frequencies and transient irregular activities of the imaged comb rows. Therefore, a main criterion for selecting animals to analyze was a consistent sign of geotaxis (up or down mood, not switching) during testing at all four orientations to gravity in both body planes of tilting (sagittal and tentacular).

Beat frequency of a comb row was determined by following the upright stage of the beat cycle (midway through the effective stroke) of a single comb plate (Fig. 2). The number of video fields required for 10 successive beats of the selected plate was counted. Slight variations between the actual beat rate and the fixed video field rate were usually compensated for over 10 successive beats, with a maximum possible counting error of one video field.

Independent counts (usually 7-10) of the number of fields for 10 beats were made at approximately regular intervals during the 1-1.5-min observation period at each tilt orientation. In order to maximize the response for all animals, the five "fastest" counts (smallest number of fields per 10 beats $=$ highest frequencies) were selected for conversion to $\mathrm{Hz}$ and calculation of the mean beat frequency and standard deviation at each tilt orientation for sagittal versus tentacular planes of tilting. In all cases, a possible one-field error in field counts was less than the standard deviations of mean beat frequencies.

\section{Isolated statocyst tilting}

Adult animals (4-4.5 cm along the aboral-oral axis) were placed individually in a Sylgard-floored glass fingerbowl of seawater on a transmitted light stage and pinned in the sagittal plane. Two parallel transverse sections across the body were cut with a dissecting scissors aboral and oral to the statocyst region. The excised section was transferred into a small Sylgard dish and oriented with the aboral surface up for further dissection under a stereo microscope. A 2-3 mm-thick flat slice containing the statocyst was cut and trimmed with dissecting and iridectomy scissors along the sagittal or the tentacular plane, depending on the desired plane of tilting of the statocyst. Sharp differential interference contrast (DIC) imaging of the statolith and balancers was difficult to achieve, even when one side of the slice (the future upper surface on the slide) was cut very close to the statocyst to remove obscuring tissue.

The tissue slice was transferred into one side of a doublechamber microscope slide ridged with Dow Corning vacuum grease. This chamber was connected to the other (overflow) chamber by a short segment of glass capillary. A $22 \times$ 

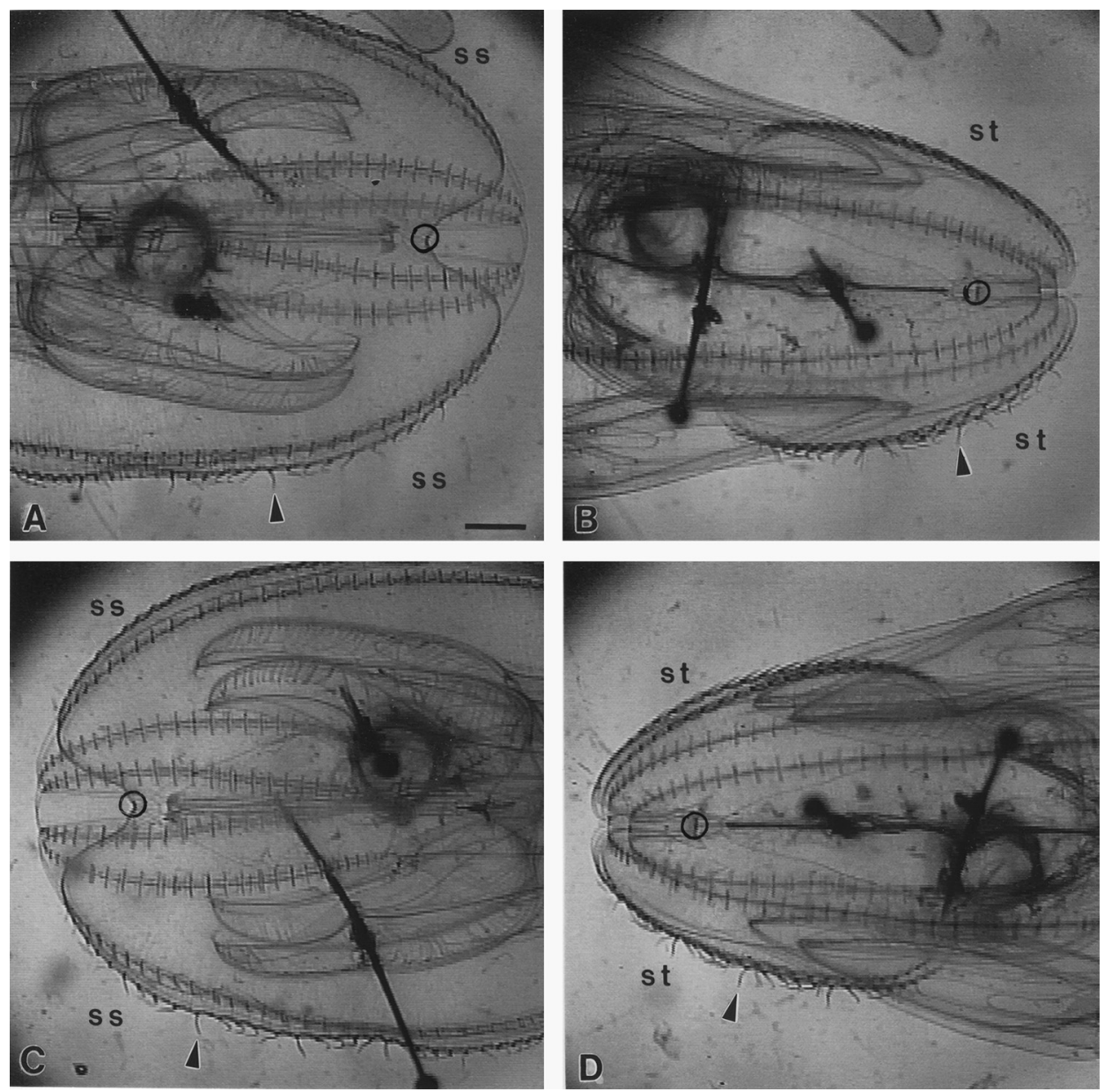

Figure 2. Motor responses of comb rows in a negatively geotactic Mnemiopsis in the cteno-tilter. The animal is pinned to a Sylgard disk and rotated in a vertical plane under seawater (see Materials and Methods). The end of the glass rod axle in the center of the disk is visible behind the animal. The location of the statocyst near the aboral end is indicated by an open circle. The mouth and oral lobes are outside the field. The animal is pinned in the sagittal plane in the left half (column) at $90^{\circ}$ (A) and at $270^{\circ}(\mathrm{C})$. The longer subsagittal comb rows (ss) are in profile view, and the lowermost rows beat actively in metachronal waves, which travel orally. Arrowheads mark single comb plates performing an effective stroke toward the aboral end. Comb plates in the uppermost subsagittal rows do not beat, but lie at rest pointing orally. In the right half (column), the same animal is pinned in the tentacular plane at $90^{\circ}$ (B) and $270^{\circ}$ (D). The shorter subtentacular comb rows (st) are in profile view, and the lowermost rows beat in metachronal waves traveling orally. Arrowheads indicate comb plates in an effective stroke. The uppermost subtentacular rows are inactive. This method was used to compare differences between beat frequencies of the activated lowermost comb rows in the same animal when tilted in the sagittal or tentacular plane at $90^{\circ}$ (A vs. B) and at $270^{\circ}$ (C vs. D). Scale bar, $0.25 \mathrm{~cm}$.

22-mm coverslip was lowered over the flat upper side of the tissue slice in the first well and gently compressed to seal the preparation and expel excess seawater into the open overflow chamber from which it was removed. The leakproof slide was then mounted on a vertical rotating stage of a horizontal Zeiss Universal microscope (micro-tilter; Tamm, 1980, 1982). An isolated living statocyst could be tilted in any orientation with respect to the direction of gravity in the sagittal or the tentacular plane. However, unlike the experiments with whole animals in a cteno-tilter, the body plane in which an isolated statocyst was tilted could not be switched, but was determined by the orientation of the dissection.

A $16 \times / 0.35$ DIC objective with the same video equipment used for whole-animal tilting was used to image micro-tilting of isolated statocysts.

\section{Analysis}

Changes in gravity-dependent displacements of the statolith and resulting deflections of the balancers at different orientations of the statocyst were determined by tracings 
from a monitor and video prints of selected still-field images. At vertical orientations $\left(0^{\circ}, 180^{\circ}\right)$, the center of the statolith lies on the oral-aboral axis on the midline of the statocyst cavity, halfway between opposing balancers. At the two horizontal orientations $\left(90^{\circ}, 270^{\circ}\right)$, the downward displacement of the statolith was measured relative to this midline reference, which was drawn halfway between the bases of the upper and lower balancers and normal to the epithelial floor for either body plane. The length of the statolith's major a-axis (if tilted in the tentacular plane) or minor b-axis (if tilted in the sagittal plane) was measured, and that part of the axis below the midline-which never exceeded either axis - was divided by the full length of the major or minor axis to give the relative (percent) downward displacement of the statolith due to gravity. Since at a vertical position without lateral displacement $\left(0^{\circ}\right.$ or $\left.180^{\circ}\right)$, $50 \%$ of the length of the major or minor axis of the statolith lies on either side of the midline, a null value of $50 \%$ was subtracted from the observed percent downward displacement of the statolith. The percent downward displacement of the statolith at $90^{\circ}$ and $270^{\circ}$ for sagittally and tentacularly tilted statocysts was averaged and plotted as a function of the plane of tilting.

The deflections of lower balancers due to downward statolith displacement at $90^{\circ}$ and $270^{\circ}$ orientations were quantified and compared in the sagittal versus tentacular planes of tilt by measuring the downward distance from the base of a balancer to its distal insertion into the statolith. This method is independent of the approximately three times greater spacing between upper and lower balancers in the tentacular versus sagittal plane.

\section{Statistical tests}

The statistical significance of differences between sample means was evaluated in two ways. Differing degrees or classes of significance were estimated in populations of animals whose mean beat frequencies of the lowermost rows were higher, but by considerably different amounts, in either the sagittal or the tentacular plane. The standard deviation for the distribution of sample means, or the standard error of the difference between the means, was used to compare relative significances. A difference of three or more standard errors between the mean beat frequencies for each plane was classed as definitely significant; a difference of more than two standard errors was regarded as probably significant; and a lesser difference, as not significant (Moroney, 1956). This treatment provided a more informative and complete account of the relative significance of the data than a "significant or not significant" test using a $P$-value. Conventional two-tailed $t$-tests were used for other statistical evaluations.

\section{Mechanical model}

A weight-and-springs mechanical simulation of the statocyst was built to help interpret the data on living statocysts. The statolith is represented by a $6.3-\mathrm{cm}$-long ovoidal piece of Styrofoam sprayed with small, 2-3 mm Styrofoam beads. The beads somewhat resembled the aggregated lithocytes of living statoliths, but were much smaller relative to statolith size. The mass (load) of the lightweight statolith was set by nails placed in the center along the major axis.

The four balancers were represented by wire coil springs. The ends of the springs were attached to the statolith and to a flat base representing the epithelial floor of the statocyst. The geometric positions of spring attachment to both the statolith and the base corresponded to the structural asymmetry of the living statocyst with respect to sagittal and tentacular body planes. The model was tilted at different orientations to gravity in the sagittal and tentacular planes and photographed with an Olympus FE-170 digital camera.

\section{Results}

\section{Whole-animal tilting}

All of the animals that displayed a consistent sign of geotaxis during the tilting experiments and were used for analysis of beat frequencies were negatively geotactic. This finding is not surprising, since ctenophores were collected at the water surface during fairly calm weather, and were predominantly in an up mood when selected for this study.

At the initial orientation $\left(0^{\circ}\right)$ of a negatively geotactic Mnemiopsis in the cteno-tilter with the oral-aboral axis vertical and the aboral pole (statocyst) facing up, only occasional waves of beating were seen on all eight comb rows, as described previously for Pleurobrachia and Beroe in an up mood (Tamm, 1980, 1982). Rotating the animal clockwise to $90^{\circ}$ with the oral-aboral axis horizontal and the aboral pole facing to the right activates fast beating of only the lowermost four comb rows (Fig. 2, top row). Since the power stroke of comb plates is directed aborally (in the opposite direction to the antiplectic metachronal waves), this asymmetric pattern of activity of the lower versus upper rows would cause a free animal to turn with its mouth facing upward (Tamm, 1980, 1982, 2014a). Turning the animal to $180^{\circ}$ with the oral-aboral axis vertical and the aboral pole facing down results in fast beating of all rows (not shown), which would cause a free animal to swim upward. Finally, at the $270^{\circ}$ orientation with the oral-aboral axis horizontal and the aboral pole facing to the left, only the lowermost four rows (formerly the uppermost rows at $90^{\circ}$ ) beat fast and the uppermost rows are inhibited (Fig. 2, bottom row), again causing a free animal to turn mouth upward.

This pattern of selective, gravity-dependent excitation and inhibition of comb row beating is characteristic of negative geotaxis (Tamm, 1980, 1982, 2014a), and occurs 
regardless of the body plane (sagittal or tentacular) in which an animal is tilted (Fig. 2). For animals pinned in the sagittal plane, comb plates in the longer subsagittal rows were in profile view and their beat frequencies were recorded (Figs. $1,2)$. For animals pinned in the tentacular plane, comb plates in the shorter subtentacular rows were in profile view so their beat frequencies were recorded (Figs. 1, 2). Since each balancer controls the beating of one subsagittal row and one subtentacular row, the motile responses of all four balancers to the direction of gravity, as expressed by comb row activity, were evident regardless of the plane of tilting.

The beat frequency activation response of the lowermost comb rows at $90^{\circ}$ and $270^{\circ}$ body orientations was therefore compared as a function of the plane, sagittal or tentacular, in which the animal was tilted. Theoretically, the beat frequencies of the lowermost rows at $90^{\circ}$ and $270^{\circ}$ should be similar (Tamm, 1982). Both orthogonal orientations to gravity were used as controls for possible internal variations or abnormalities in individual animals, and were then discarded.

There was no significant effect on recorded beat frequencies related to which body plane was tested first in the cteno-tilter. Therefore, the data for both orders of testing were combined into one set for tilting in the sagittal plane or the tentacular plane.

At a $90^{\circ}$ body orientation, the mean beat frequency of the lowermost comb rows was higher when an animal was tilted in the sagittal plane in 17 of the 21 animals analyzed (81\%) (Fig. 3). However, there were considerable variations from animal to animal between the sample means in the two planes. The number of standard errors of the difference between means was used to judge their relative significances (see Materials and Methods). In the 17 animals noted above, the difference between the mean beat frequency for each plane was definitely significant in 9 animals, probably significant in 2 animals, and not significant in 6 animals (Fig. 3). At the same $90^{\circ}$ orientation, the mean beat frequency of the lowermost rows was higher in the tentacular plane in 4 of the 21 animals (19\%). In these animals, the difference between the mean beat frequency for each plane was probably significant in 2 animals, but not significant in the other 2 animals (Fig. 3).

At the other orthogonal orientation $\left(270^{\circ}\right)$, a similar but less pronounced pattern was found. The mean beat frequency of the lowermost rows was higher when an animal was tilted in the sagittal plane for 15 of the 21 animals $(71 \%)$. In these animals, the difference between the mean beat frequency for each plane was definitely significant in 4 animals, probably significant in 1 animal, and not significant in 10 animals (Fig. 3). At the same $270^{\circ}$ orientation, the mean beat frequency of the lowermost rows was higher in the tentacular plane in 6 of the 21 animals (29\%). In these

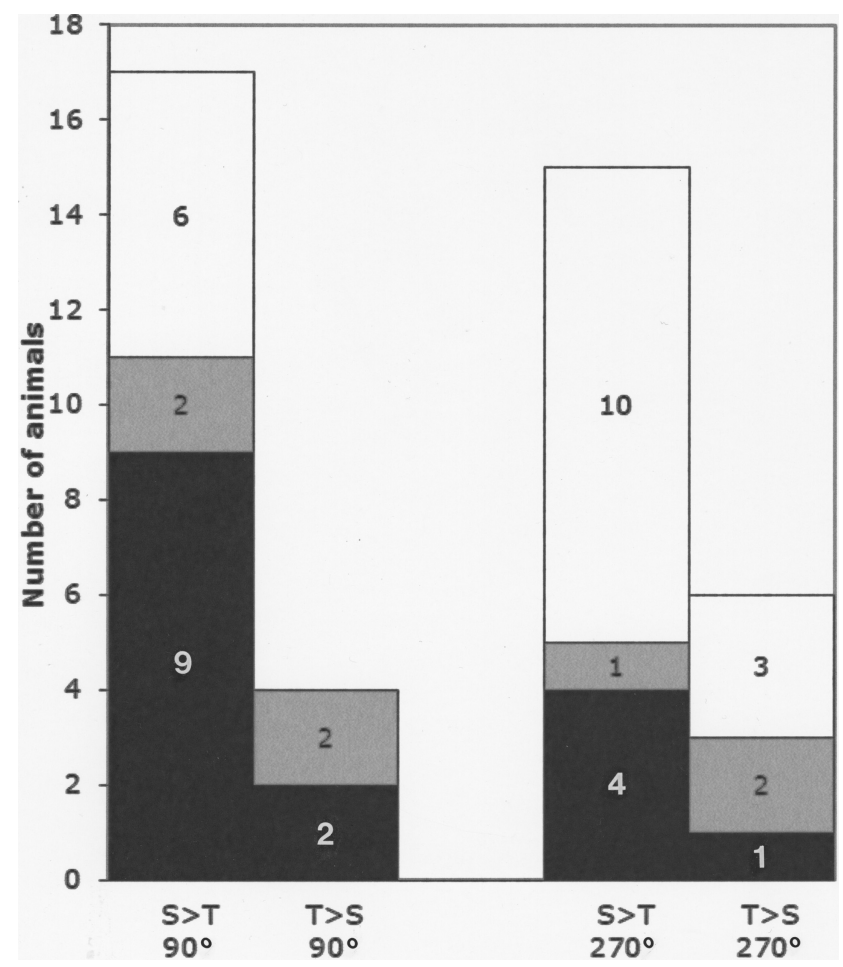

Figure 3. Comparative distribution of numbers of animals whose mean beat frequencies of the lowermost comb rows were higher when an animal was tilted in the sagittal plane than in the tentacular plane $(\mathrm{S}>\mathrm{T})$ or vice versa $\left(\mathrm{T}>\mathrm{S}\right.$ ) at $90^{\circ}$ (left column pair) and $270^{\circ}$ (right column pair). Twenty-one animals were tested at the two orthogonal orientations in both body planes. The total number of $\mathrm{S}>\mathrm{T}$ animals and $\mathrm{T}>\mathrm{S}$ animals in each column was subdivided into the number of animals in three different significance classes: definitely significant (black), probably significant (gray), and not significant (white) (based on standard errors of the difference; see Materials and Methods).

animals, the difference between the means was definitely significant in 1 animal, probably significant in 2 animals, but not significant in the remaining 3 animals (Fig. 3).

In the smaller number of cases where the mean beat frequency of the lowermost rows was higher in the tentacular plane at $90^{\circ}$ or $270^{\circ}$, the mean beat frequency of the lowermost rows at the other orthogonal orientation of the same animal $\left(270^{\circ}\right.$ or $\left.90^{\circ}\right)$ was higher in the sagittal plane in all but one animal, in which the mean beat frequency of the lowermost comb rows of that animal was higher in the tentacular plane at both $90^{\circ}$ and $270^{\circ}$ orientations.

Figure $4 \mathrm{~A}-\mathrm{C}$ shows three examples at $90^{\circ}$ orientation of significantly higher mean beat frequencies of the lowermost rows when animals were tilted in the sagittal plane (taken from the 9 cases in Fig. 3). In Figure 4A, the mean beat frequency in the sagittal plane was $24 \mathrm{~Hz}$ compared with $18 \mathrm{~Hz}$ in the tentacular plane, a $33 \%$ difference. Figure 4D shows significantly higher mean beat frequencies of the lowermost rows in the sagittal plane at both $90^{\circ}$ and $270^{\circ}$ orientations of the same animal, with similar beat frequencies noted in either body 


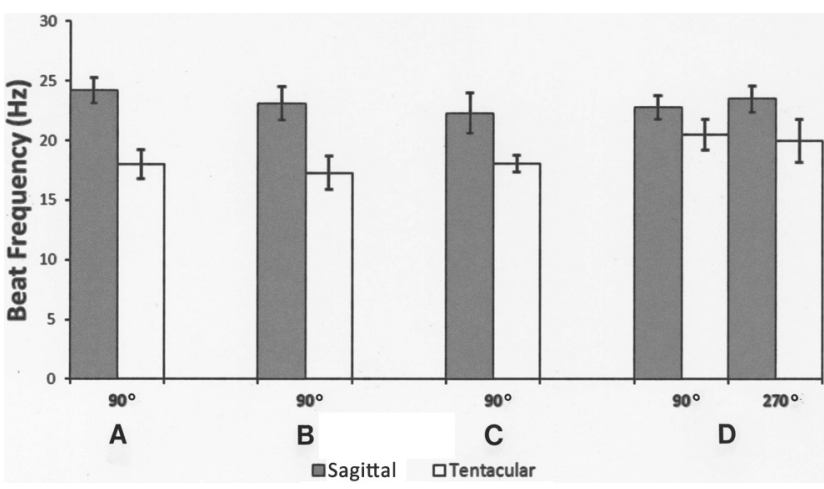

Figure 4. Individual examples of significantly higher mean beat frequencies of the lowermost rows in the sagittal plane (gray) and the tentacular plane (white) at $90^{\circ}$, for four different animals (A-D), including one animal at both $90^{\circ}$ and $270^{\circ}$ orientations (D). Note that in this animal, the higher beat frequencies in the sagittal plane are similar at both $90^{\circ}$ and $270^{\circ}$, as are the lower beat frequencies in the tentacular plane at these orientations (D). Error bars are standard deviations.

plane at both orthogonal orientations. For all sagittal versus tentacular plane pairs shown, $P<0.01$ except at $270^{\circ}$ in Figure 4D, where $P<0.05$.

\section{Isolated statocyst tilting}

To interpret the differences in comb row activity at $90^{\circ}$ and $270^{\circ}$ for animals tilted in the sagittal or the tentacular plane, I examined the relative downward displacement of the statolith and resulting deflections of the mechanoresponsive balancers with respect to the orthogonal body planes. Tissue slices containing the statocyst were dissected in the sagittal or the tentacular plane, mounted on leakproof slides, and rotated to different orientations with respect to gravity on a vertical rotating stage of a horizontal microscope (micro-tilter). Due to variations in dissection and tissue orientation on the slide, many preparations did not allow satisfactory imaging of the statolith and balancers, and were discarded. Of the slides used, the balancers nearest the sagittal or tentacular surface of the slice were the clearest. Because the statolith and balancers reside in slightly different focal planes when viewed laterally, both structures usually were not completely in focus, and required throughfocus imaging for analysis.

At the two vertical orientations to gravity of isolated statocyst preparations, corresponding to $0^{\circ}$ and $180^{\circ}$ orientations of whole animals in a cteno-tilter, statolith position and balancer configurations were oppositely and symmetrically affected by gravity, and in a similar manner in both the sagittal and tentacular planes (Fig. 5).

The superellipsoidal statolith was located with its center on the oral-aboral axis at the intersection of the sagittal and tentacular planes (i.e., on the midline of the epithelial floor in either plane; Fig. 5). At these orientations, all four balancers experience equal loading or unloading by the statolith, and usually show the same beat frequency, which depends on the animal's mood (see Tamm, 1980, 1982).

At $0^{\circ}$ (mouth downward, statocyst upward in intact animals), the statolith rested close to the epithelial floor, against the bent bases of the balancers (Fig. 5A, B). Since only the basal part of a balancer actively moves during a beat (Tamm, 1980, 1982), the beating of balancers at $0^{\circ}$ transiently lifts the statolith slightly away from the epithelial floor. The distal regions of the balancers were closely curved around the sides of the statolith near their entrance into it. At $180^{\circ}$ (mouth upward, statocyst downward in intact animals), the statolith hung some distance down from the epithelial floor at the straightened distal ends of the balancers (Fig. 5C, D).

At the two horizontal orientations to gravity of an isolated statocyst, corresponding to $90^{\circ}$ and $270^{\circ}$ positions of whole animals in a cteno-tilter, the statolith was displaced downward from its equilibrium position at the center of the statocyst cavity (Fig. 6). The lower (activated) balancer was pushed away from the midline by the load of the statolith, and the upper (inhibited) balancer was pulled toward the midline (Fig. 6). In both cases, the weight of the statolith bent only the curved distal region of the balancers, but their motile responses occurred at the base (Tamm, 1980, 1982).

Figure 7 illustrates the geometric methods used for quantitating and comparing statolith displacements and the resulting balancer deflections at orthogonal orientations $\left(270^{\circ}\right.$ shown) in both sagittal and tentacular planes (see Materials and Methods).

Measurements of isolated statocysts in slices prepared in the sagittal plane $(n=11)$ or in the tentacular plane $(n=9)$ show that the mean statolith displacement from the midline at $90^{\circ}$ is similar to that at $270^{\circ}$ in either body plane. However, the mean statolith displacements at $90^{\circ}$ and $270^{\circ}$ are about $5 \%$ greater in statocysts tilted in the sagittal plane $(\sim 30 \%)$ than in the tentacular plane $(\sim 25 \%)$ (Fig. 8). This difference between statolith displacements in the two body planes is significant by three standard errors with a $P$-value $<0.01$.

The downward deflections of the lowermost balancers at $90^{\circ}$ and $270^{\circ}$ were analyzed because intact animals in the cteno-tilter were negatively geotactic with fast beating of the lowermost comb rows at these orientations. Since these balancers were more clearly imaged at $270^{\circ}$ than at $90^{\circ}$ tilt, the deflections at $270^{\circ}$ orientation were analyzed in the two body planes (Fig. 7). The mean downward deflection of the distal region of a lower balancer at $270^{\circ}$ was $42 \pm 3.8 \mu \mathrm{m}$ $(n=5)$ in the sagittal plane and $28 \pm 3.0 \mu \mathrm{m}(n=8)$ in the tentacular plane $(P<0.01)$.

Thus, at a right-angle orientation to gravity, an approximately 1.2 times greater downward displacement of the statolith in the sagittal than in the tentacular plane is 

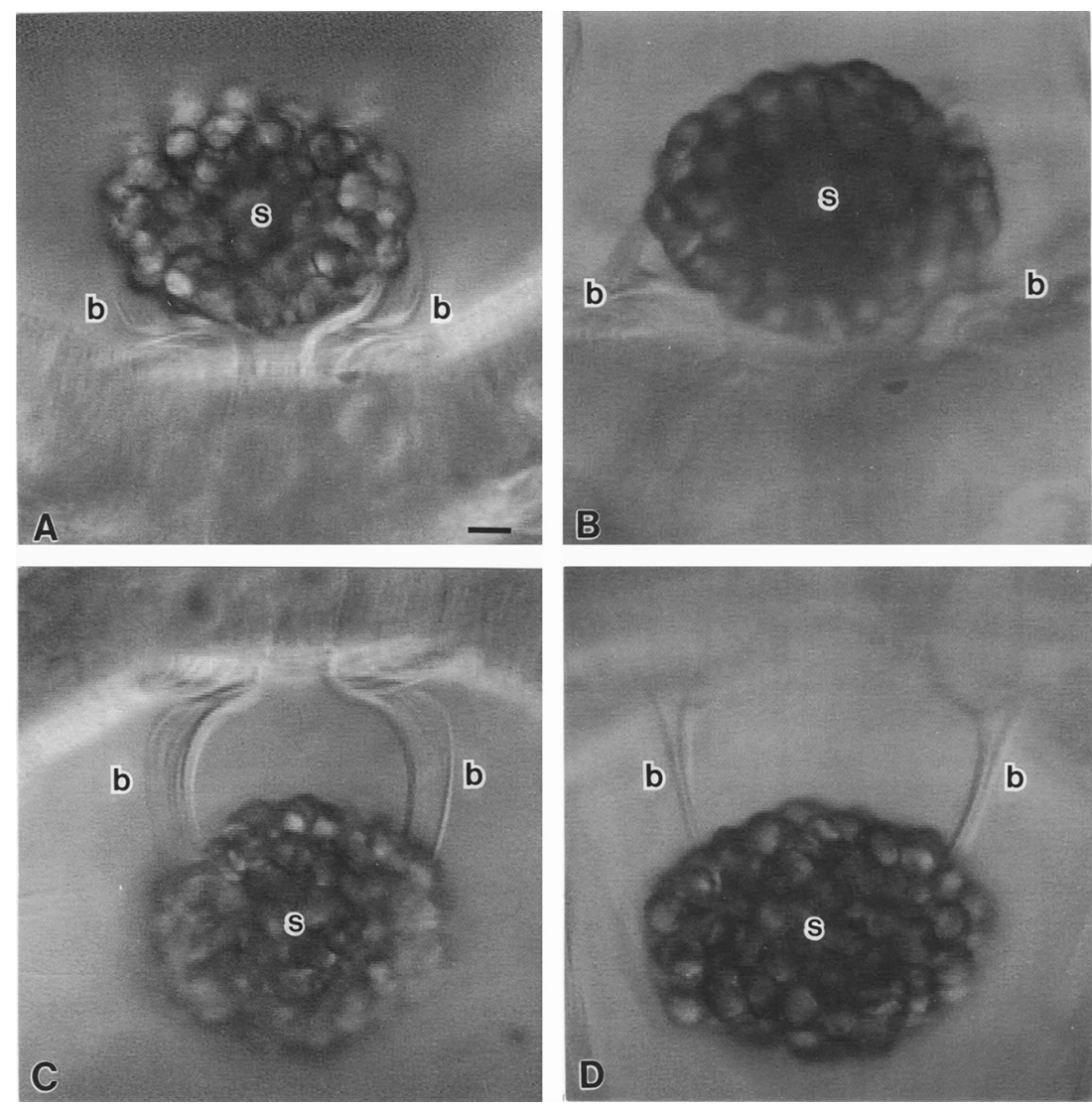

Figure 5. Micro-tilting of isolated statocysts at vertical orientations in the sagittal versus tentacular planes at $0^{\circ}$ (A vs. B) and $180^{\circ}$ (C vs. D). The four images are arranged with sagittal views in the left column and tentacular views in the right column, with the same orientation to gravity on the left and right for comparisons between the two planes. Note that the bases of the balancers (b) are set closer together in the sagittal plane $(\mathrm{A}, \mathrm{C})$ than in the tentacular plane $(\mathrm{B}, \mathrm{D})$ at both orientations. In $\mathrm{B}$, the compressed balancers are partially obscured. The two orientations in the sagittal plane are from the same animal, and those in the tentacular plane are from a different animal. Scale bar, $10 \mu \mathrm{m}$.

correlated with about a 1.5 times greater downward deflection of the lowermost balancers between these two planes.

The sign of geotaxis of isolated statocyst slices in the micro-tilter was more variable within a preparation and between different slides compared with intact animals used in the cteno-tilter, perhaps due to dissection, absence of the rest of the animal's body, microscopy conditions, or other factors. Because of these variations, the beat frequency responses of balancers in isolated statocysts could not reliably be compared between the sagittal and tentacular planes.

\section{Mechanical model}

The model statocyst in a vertical orientation to gravity $\left(0^{\circ}\right.$ in living statocysts) presented its Styrofoam statolith with its center on the midlines of the sagittal and tentacular planes, and with similar configurations of all four supporting balancer springs (Fig. 9A). At a horizontal orientation of the model $\left(270^{\circ}\right.$ in living statocysts), the statolith was displaced downward by gravity a greater distance from the midline when tilted in the sagittal plane than in the tentacular plane (Fig. 9B vs. C). This result was due entirely to geometry, that is, the elongated distribution of load supported on a rectangular arrangement of flexible cantilevers. The degree of statolith displacement in each plane was remarkably similar to that found in living, isolated statocysts (Figs. 6, 8), due to trial-and-error selection of an appropriate weight for a statolith resting on the wire coil springs used in the model. The deflection of the lowermost balancer springs was therefore greater when the model was tilted in the sagittal plane (Fig. 9B, C), similar to the findings in living statocysts (Fig. 6). The significance of the model for understanding the behavior of the living statocyst is discussed below. 

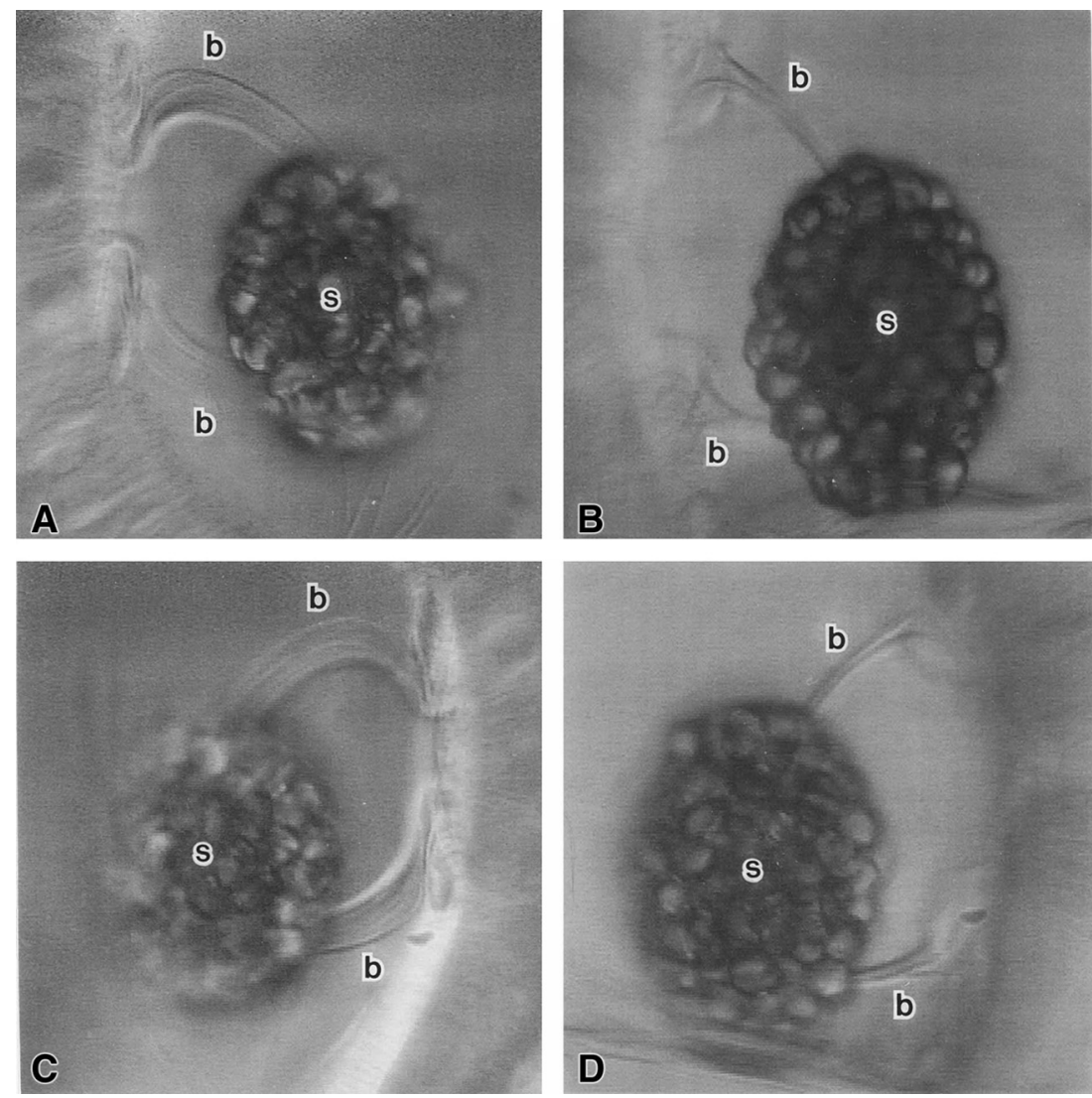

Figure 6. Micro-tilting of isolated statocysts at horizontal orientations in the sagittal and tentacular planes at $90^{\circ}$ (A vs. B) and $270^{\circ}$ (C vs. D). The four images are arranged with sagittal views in the left column and tentacular views in the right column, with the same orientation to gravity on the left and right for comparisons between the two planes. Note that the bases of the balancers (b) are closer together in the sagittal plane (A, C) than in the tentacular plane (B, D) at both orientations. The two orientations in the sagittal plane are from the same animal, as in Fig. 5A, C, and those in the tentacular plane are from the same animal, as in Fig. 5B, D. Magnification is the same as in Figure 5.

\section{Discussion}

This study reports the functional consequences of a recently discovered design asymmetry in the geotactic organ of ctenophores (Tamm, 2014c). The architecture of the ctenophore statocyst previously had been considered to be radially symmetric about the longitudinal body axis (oralaboral axis) and in the two orthogonal planes of symmetry (sagittal and tentacular) along this axis. For this reason, previous studies on the mechanism of geotaxis, particularly the beat frequency responses of specific comb rows to angular changes in body orientation to gravity, as seen in a cteno-tilter (Tamm, 1980, 1982, 2014), did not take into account (or alter) the body plane in which an animal was tilted. For example, cydippids like Pleurobrachia, the most radially symmetric ctenophores with nearly equal sagittal and tentacular axes, were routinely tested in the tentacular plane. In comparison, beroids (Beroe) and lobates (Bolinopsis) were commonly tilted in their flattened sagittal or stomodeal plane; the results in both planes were treated equivalently (Tamm, 1980, 1982, 2014a).

The discovery of a bilateral morphological asymmetry of the statocyst with respect to the sagittal and tentacular planes (Tamm 2014c) prompted the present investigation into whether this newly revised structural plan affects the geotactic responses of ctenophores. I report here that it does: function follows structure, as Crick (1988) suggested, also in the statocyst of ctenophores. The cteno-tilting experiments on whole animals in an up mood convincingly show that the activated lowermost comb rows at orthogonal orientations to gravity beat faster when an animal is tilted in the sagittal plane than in the tentacular plane (Fig. 3). Although the differences in mean beat frequencies between the two body planes of an animal varied widely and ranged from insignificant to highly significant, a trend for an increased motile response of the lowermost comb rows in the sagittal plane was clear (Figs. 3, 4). Although animals in a down mood were not available for testing, the same results 


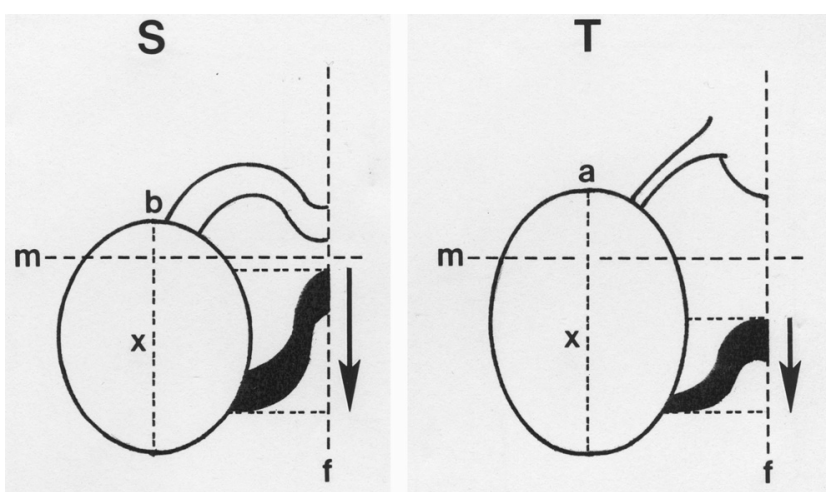

Figure 7. Protocol for determining statolith displacements and balancer deflections due to gravity in isolated statocysts at orthogonal orientations $\left(270^{\circ}\right.$ shown). In the sagittal plane $(\mathrm{S})$, the minor b-axis of the superellipsoidal statolith is parallel to the gravity vector (down) and the balancers are closely spaced. In the tentacular plane (T), the major a-axis of the statolith is parallel to gravity and the balancers are farther apart (see Figs. 1, 5, 6). The relative downward displacement of the statolith from the midline $(\mathrm{m})$ of the epithelial floor (f) was calculated as the length of the axis below the midline ( $\mathrm{x}$ ) divided by the full length of the axis ( $\mathrm{b}$ or a) as a percent, and subtracting $50 \%$ (the equilibrium value at vertical orientations). The downward deflection of a lowermost balancer (black) at $90^{\circ}$ and $270^{\circ}$ was measured as the vertical distance along the floor from its base to its entrance into the statolith (dashed lines and arrow). (See Materials and Methods).

are assumed for positively geotactic animals, except that the uppermost, not lowermost, comb rows would be activated at $90^{\circ}$ and $270^{\circ}$ orientations (see Tamm, 1980, 1982, 2014a).

Micro-tilting of isolated statocysts in tissue slices confirmed expectations from whole-animal tilting: that both the amount of downward displacement of the statolith and the degree of deflection of the lowermost balancers by the load of the statolith are greater in the sagittal plane. Thus, the differences in comb row beat frequencies between the two body planes is correlated with a greater gravitational stimulus of the sensory-effector organ (statocyst) controlling geotaxis.

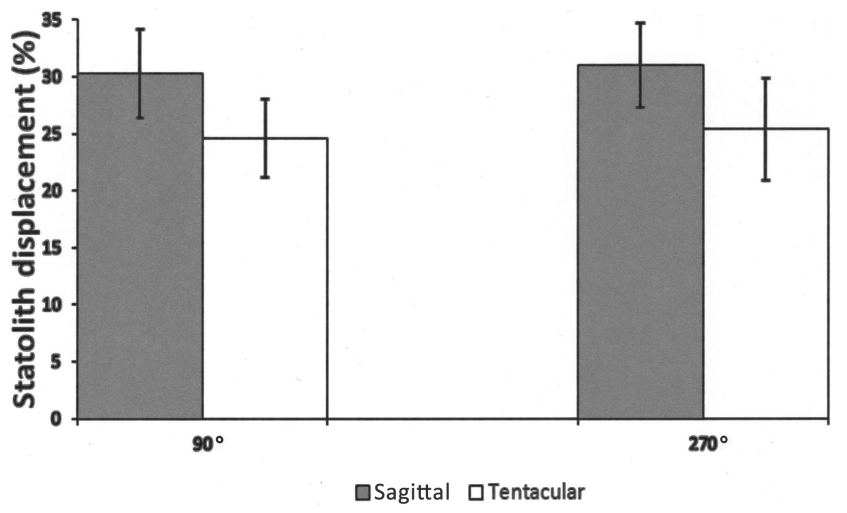

Figure 8. Relative statolith displacements at $90^{\circ}$ and $270^{\circ}$ in isolated statocysts tilted in sagittal (dark) or tentacular (white) planes. Y-bars are standard deviations.
The approximately 1.2 times greater downward displacement of the statolith in the sagittal plane results in about a 1.5 times greater downward deflection of the lowermost balancers in the sagittal plane, implying a non-linear stress-strain relationship between applied load and ciliary deformation. Previous micromanipulation experiments showed that the beat frequency of isolated, statolith-free balancers of Mnemiopsis increased in a linear fashion with increasing amounts of deflection by a suction-pipette attached to the ciliary tips (Lowe, 1997). Since I was not able to quantify the effects of differing degrees of statolith displacement and balancer deflection on the beat frequency of a balancer, the stimulus-response relationship of balancers in situ remains a topic for future work.

However, it seems clear from the whole-animal experiments that the greater downward deflection of the lowermost balancers in isolated statocysts tilted in the sagittal plane causes a higher beat frequency of these balancers with correspondingly higher rates of beating of the lowermost comb rows, as observed in the sagittal plane using the cteno-tilter.

\section{Explanation of results}

The similarities between the mechanical model (Fig. 9) and living statocysts in the different amounts of statolith displacement and balancer deflection when tilted in sagittal versus tentacular planes offers important clues for interpreting the results.

The wire helical springs (balancers) have a similar resistance to lateral flexion regardless of the direction of applied force (i.e., statolith load in the sagittal or tentacular plane). The observed differences in statolith displacement and balancer deflections between the two body planes in the model thus reflect the physical asymmetries of its design: four cantilever balancers are located at the vertices of a rectangle lying in the tentacular plane, which are end-loaded by an ovoidal gravitational mass with its long axis also oriented in the tentacular plane. This matching geometric asymmetry of load and supporting elements is sufficient by itself to account for the difference in mechanical and motile outputs observed between tilting living statocysts and ctenophores in sagittal versus tentacular planes.

Of course, a model (or theory) that is consistent with the data does not necessarily mean it is correct, or even a complete explanation. The most important simplification of the model is the nature of the balancers. Living balancers, unlike coil springs, are not cylindrical in shape with a uniform diameter (even though balancers are commonly depicted as such in diagrams like Fig. 1). Instead, the base of a balancer is flared into a wide $\mathrm{V}$, with the cilia arranged in rows forming a rhomboidal lattice (Tamm, 1982; Tamm and Tamm, 2002). About one third of the 150-200 cilia in a balancer diverge from the main group near the base and overlap the bent distal ends of cilia at the beginning of a subsagittal ciliated groove (Tamm, 1982). The majority of 

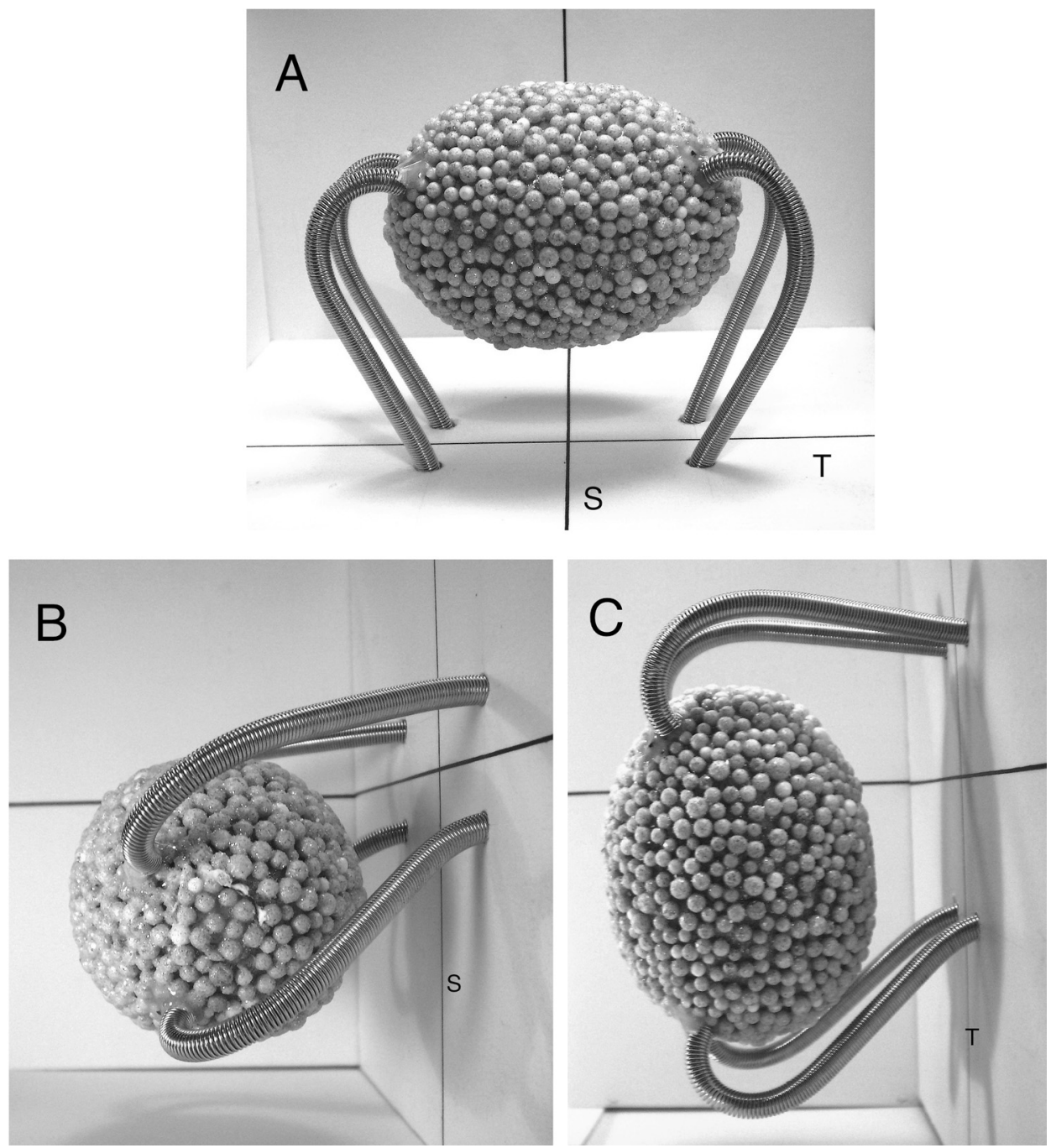

Figure 9. Mechanical model of the statocyst viewed at $0^{\circ}$ in the tentacular plane (A), tilted to $270^{\circ}$ in the sagittal plane (B), and tilted to $270^{\circ}$ in the tentacular plane (C). At $0^{\circ}$, the ovoidal, beaded Styrofoam statolith and the four balancer springs are symmetrically disposed about the midlines (black lines) of the sagittal (S) and tentacular (T) planes (see Fig. 5). Note that the four balancers are arranged in a rectangle parallel to the tentacular plane and the longer axis of the statolith (see Fig. 1). At $270^{\circ}$, the statolith is displaced farther downward from the midline in the sagittal plane (B) than in the tentacular plane (C). As a result, the lowermost balancers are deflected a greater distance from the midline in the sagittal plane (see Fig. 6).

the balancer cilia that curve upward toward the statolith appear differently in the two body planes: as a broad ribbon when viewed in the sagittal plane, and as a narrow, tapering stalk when observed in the tentacular plane (Figs. 5, 6, 7). Therefore, the balancer shaft, which is deflected under load, is not cylindrical like a coil spring, but is shaped like a strip flattened in the sagittal plane and has a different crosssectional profile in each body plane. However, the midlines of the V-shaped bases of the balancers are not oriented preferentially with respect to either the sagittal or tentacular plane, but lie diagonally to each plane; that is, their orientation is geometrically equivalent in both body planes.

Any or all of these known features of balancer structure and orientation might affect their deformation under a load directed in a given plane. Future work should elucidate the importance of the geometric asymmetries of load and balancer arrangement, as exemplified by the model, in relation to the structural properties of real balancers for understanding the observations reported here.

\section{Possible adaptive value}

Since the observed differences in ciliary beat frequencies are likely to arise largely from the asymmetric design of the statocyst, one may ask first whether this architectural constraint is itself an adaptation for an essential function, or 
exists only as a byproduct of other processes or factors (Gould and Lewontin, 1979). If the latter is the case, then these functional findings may not have any adaptive value in the life of ctenophores. A more relevant question is whether, in free, unrestrained animals turning to swim up or down, the effectiveness of the righting response depends on the plane in which an animal is tilted. If the righting response of animals in nature is indeed stronger in the sagittal than the tentacular plane, then one may ask if this behavior is advantageous for ctenophores, and, if so, how.

One approach to this question is to try to rule out possible candidates, such as a benefit related to asymmetric body shape. In lobate ctenophores like Mnemiopsis, the body is flattened in the tentacular plane and expanded along the sagittal plane (Fig. 1). For Mnemiopsis in a vertical position in the water (at $0^{\circ}$ or $180^{\circ}$ ), an approaching directional disturbance (wave or current) is more likely to have a greater impact encountering the broader sagittal surface than the narrower tentacular side, thereby causing an animal to be tilted more often and to a greater extent in the tentacular plane. A stronger righting response in the tentacular plane would therefore be expected to be more advantageous for regaining vertical balance. But the opposite response was found, implying that these results would be disadvantageous by this hypothesis.

A further argument against a body shape-related, adaptive value is the well-known diversity of body plan symmetry in different orders of ctenophores. Cydippids, like Pleurobrachia, are almost radially symmetric, with nearly equal sagittal and tentacular axes. Yet their statocyst shares the same structural asymmetries with respect to the two body planes as the statocyst of Mnemiopsis (Tamm, 2014c). If function follows structure in the statocyst of different ctenophores, I would expect to find a higher beat frequency in the sagittal plane of tilted Pleurobrachia, as reported here for Mnemiopsis. This theory has not been tested.

In conclusion, testable predictions from other proposed adaptive "reasons" for my findings clearly await better hypotheses based on a more extensive knowledge of the behavior of ctenophores in the sea.

\section{Acknowledgments}

I thank James L. Olds for asking the question that initiated this investigation. I am grateful to Benjamin Moran and Shannon Jones of the Bay Paul Center, MBL, for their generous assistance in preparing column charts with error bars; to David Mark Welch, Director of the Bay Paul Center, for significant advice on statistics; to Susan Banks of the Bell Center for her expert help in scanning figures; and to Mark Terasaki for discussions and support. Signhild Tamm spotted the ideal item to use for the statolith in the model.

\section{Literature Cited}

Aronova, M. 1974. Electron microscopic observations on the aboral organ of ctenophores. Z. Mikrosk. Anat. Forsch. 88: 401-412.

Budelmann, B. U. 1988. Morphological diversity of equilibrium receptor systems in aquatic invertebrates. Pp. 757-782 in Sensory Biology of Aquatic Animals, J. Atema, R. R. Fay, A. N. Popper, and W. N. Tavolga, eds. Springer-Verlag, New York.

Chun, C. 1880. Die Ctenophoren des Golfes von Neapel und der angrenzenden Meeres-Abschnitte. Flora und Fauna des Golfes von Neapel, Vol. 1. Engelmann, Leipzig.

Crick, F. 1988. What Mad Pursuit. A Personal View of Scientific Discovery. Basic Books, New York. 182 Pp.

Gould, S. J., and R. C. Lewontin. 1979. The spandrels of San Marco and the Panglossian paradigm: a critique of the adaptationist programme. Proc. R. Soc. Lond. B 205: 581-598.

Horridge, G. A. 1966. Pathways of co-ordination in ctenophores. Pp. 247-266 in The Cnidaria and Their Evolution, W. J. Rees, ed. Zool. Soc. London 16. Academic Press, New York.

Horridge, G. A. 1971. Primitive examples of gravity receptors and their evolution. Pp. 203-221 in Gravity and the Organism, S. A. Gordon and M. J. Cohen, eds. University of Chicago Press, Chicago.

Horridge, G. A. 1974. Recent studies on the Ctenophora. Pp. 439-468 in Coelenterate Biology: Reviews and New Perspectives, L. Muscatine and H. M. Lenhoff, eds. Academic Press, New York.

Krisch, B. 1973. Über das Apikalorgan (Statocyste) der Ctenophore Pleurobrachia pileus. Z. Zellforsch. 142: 241-262.

Lowe, B. 1997. The role of calcium in deflection-induced excitation of motile mechanoresponsive balancer cilia in the ctenophore statocyst. $J$. Exp. Biol. 200: 1593-1606.

Moroney, M. J. 1956. Facts From Figures. Penguin Books, Baltimore, MD.

Noda, N., and S. L. Tamm. 2014. Lithocytes are transported along the ciliary surface to build the statolith of ctenophores. Curr. Biol. 24: R951-R952.

Samassa, P. 1892. Zur Histologie der Ctenophoren. Arch. Mikrosk. Anat. 40: $157-243$.

Schnitzler, C. E., K. Pang, M. L. Powers, A. M. Reitzel, J. F. Ryan, D. Simmons, T. Tada, M. Park, J. Gupta, S. Y. Brooks et al. 2012. Genomic organization, evolution, and expression of photoprotein and opsin genes in Mnemiopsis leidyi: a new view of ctenophore photocytes. BMC Biol. 10: 107.

Tamm, S. L. 1980. Cilia and ctenophores. Oceanus 23: 50-59.

Tamm, S. L. 1982. Ctenophora. Pp. 266-358 in Electrical Conduction and Behaviour in 'Simple' Invertebrates, G. A. B. Shelton, ed. Oxford University Press, Oxford.

Tamm, S. L. 2012a. Regeneration of ciliary comb plates in the ctenophore Mnemiopsis leidyi. I. Morphology. J. Morphol. 273: 109-120.

Tamm, S. L. 2012b. Patterns of comb row development in young and adult stages of the ctenophores Mnemiopsis leidyi and Pleurobrachia pileus. J. Morphol. 273: 1050-1063.

Tamm, S. L. 2014a. Cilia and the life of ctenophores. Invertebr. Biol. 133: $1-46$.

Tamm, S. L. 2014b. Ctenophores and termites-systems for motility. Pp. 147-171 in Cilia and Flagella: Ciliates and Flagellates, K. Hausmann and R. Radek, eds. Schweizerbart, Stuttgart, Germany.

Tamm, S. L. 2014c. Formation of the statolith in the ctenophore Mnemiopsis leidyi. Biol. Bull. 227: 7-18.

Tamm, S. L., and S. Tamm. 2002. Novel bridge of axon-like processes of epithelial cells in the aboral sense organ of ctenophores. J. Morphol. 254: 99-120. 